\title{
DEPENDENCIA DE LA COCAÍNA Y PSICOPATOLOGÍA A TRAVÉS DEL SCL-90-R
}

\author{
ANA LÓPEZ Y ELISARDO BECOÑA
}

Universidad de Santiago de Compostela

\begin{abstract}
Resumen: Analizar las características psicopatológicas de las personas con dependencia de la cocaína que demandan tratamiento en centros de drogodependencias es un tema relevante, dado el importante incremento de estas demandas en los últimos años. El objetivo del presente estudio es analizar la presencia de determinados síntomas psicopatológicos y el malestar percibido por el sujeto con el SCL-90-R en una muestra de 115 sujetos en tratamiento por dependencia de la cocaína. Los resultados que hemos obtenido indican que las escalas de sintomas con mayor puntuación son la de depresión, obsesión-compulsión, ideación paranoide y sensibilidad interpersonal. Un $36.5 \%$ de la muestra se situaría en la población psiquiátrica del baremo de este cuestionario. Esto sugiere la necesidad de evaluar estas características psicopatológicas para un diseño adecuado del tratamiento de estas personas, que junto a la dependencia de la cocaina tienen con frecuencia asociados otros trastornos.
\end{abstract}

Palabras clave: Cocaína, dependencia, psicopatología, SCL-90-R.

\section{Cocaine dependence and psychopathology measured with the SCL-90-R}

\begin{abstract}
The analysis of psychopathological characteristics of people with cocaine dependence demanding treatment in centers of drug abuse is a relevant topic, due to the relevant increase of these demands in the last year. The objective of the present study is to analyze the presence of certain psychopathological symptoms and the disconfort perceived by the subject with the SCL-90-R in a sample of 115 subjects in treatment for cocaine dependence. The results obtained indicate that the scales of symptoms with more punctuation are these of depression, obsession-compulsion, paranoid ideation and interpersonal sensibility. The $36.5 \%$ of the sample would be located in the psychiatric population according the norms of this questionnaire. This suggests the necessity to evaluate these psychopathological characteristics for an appropriate design of the treatment of these people wich cocaine dependence what suffer other disorders.
\end{abstract}

Key words: Cocaine, dependence, psychopathology, SCL-90-R.

\section{INTRODUCCIÓN}

La demanda de tratamiento por problemas con el consumo de cocaína continúa creciendo en España. En el año 2002 había en los Centros Públicos de Drogodependencias 11.904 personas en tratamiento por consumo de cocaína como droga principal (para el $59,8 \%$ era el primer tratamiento por problemas con el consumo de drogas) cuando en el año 1991 eran tan sólo 943. Si analizamos sólo el porcentaje de personas que demandan por primera vez tratamien-

Recibido: 9 de enero de 2006. Aceptado: 23 de febrero de 2006.

Correspondencia: Ana López Durán. Universidad de Santiago de Compostela, Departamento de Psicología Clínica y Psicobiología, Campus Universitario Sur, 15782 Santiago de Compostela. Correo-e: alduran@usc.es. to, la cocaína fue la droga que causó un mayor número de admisiones $(41,7 \%$ frente a $28,3 \%$ de heroina en 2002) (PNSD, 2005).

La cocaína, también denominada "coca», "farlopa», «nieve» o "perica» entre otras muchas acepciones, es el producto más popular (ver Tabla 1) que se extrae del arbusto de la coca (Erythroxilum coca). Es clorhidrato de cocaína que se obtiene a partir de la mezcla de la pasta de coca con acetona o éter, la solución se filtra añadiendo amoniaco y se obtiene la cocaina base. La siguiente fase es disolverla en éter etaico, y se filtra agregando ácido clorhídrico y acetona (García, 2002). Se presenta como un polvo blanco, cristalino y de sabor amargo que se puede consumir por vía nasal («esnifada»), oral, o endovenosa. También hay 
datos sobre el consumo de cocaína fumada en cigarrillos o mezclada con cannabis (Observatorio Europeo de la Droga y la Toxicomanía, 2004).

Además de los problema derivados directamente del consumo de cocaína, en los últimos años está cobrando relevancia el análisis de la presencia de problemas psicopatológicos en las personas que demandan tratamiento por problemas con el consumo de drogas. First y Gladis (1996), Ochoa (2000), Rosenthal y Westreich (1999), San (2004) Swendsen y Merikangas (2000) y Ziedonis (1992) señalan que es necesario evaluar la presencia de problemas psicopatológicos en estas personas porque implica la existencia de condiciones más crónicas, mayor resistencia al tratamiento $y$ hay un deterioro psicosocial más severo. Además, desde el punto de vista del tratamiento, los sujetos que tienen problemas con el consumo de sustancias y otro problema psicopatológico buscan ayuda profesional con más frecuencia; hay implicaciones etiológicas, ya que de la aparición simultánea de ambos trastornos se pueden derivar distintas explicaciones; respecto a las consecuencias a nivel diagnóstico y clínico, puede ser más adecuado agrupar a los consumidores en función de la patología común que presentan porque pueden requerir tratamientos distintos a los tradicionales; y la respuesta al tratamiento también puede variar, ya que presentan un mayor deterioro a todos los niveles, por lo que su pronóstico es más pobre. Concretamente en el caso de los consumidores de cocaína, Gawin y Kleber (1986) apuntan que los sujetos con trastorno psiquiátrico aunque consumen menos canti- dad de cocaína muestran un deterioro similar que los que consumen más cantidad y no tienen ningún trastorno. Además, solicitan antes tratamiento.

Como apuntan Rosenthal y Wesireich (1999) y Sánchez (2001), es necesario evaluar la presencia de síntomas psicopatológicos en todos los consumidores de sustancias que acuden a tratamiento. Además de facilitar la definición del plan de intervención en el problema con el consumo de drogas, la evaluación nos facilita tener una visión global del paciente y de sus problemas.

Entre los autoinformes utilizados en el campo de las drogodependencias para evaluar a consumidores de cocaína destaca el SCL-90 (Derogatis, 1973) y su versión posterior el SCL-90-R (Derogatis, 1977) (Brown, Seraganian y Tremblay, 1994; Castaneda, 1994; Castaneda, Galenter y Franco, 1989; Kleinman et al., 1992; Rose, Brown y Haertzen, 1989; Weiss, Mirin, Griffin y Michael, 1988; Weiss, Mirin, Michael y Sollogub, 1986). Este instrumento evalúa el malestar subjetivo, pero sin hacer una valoración diagnóstica (Derogatis, 2002). Está formado por nueve dimensiones de síntomas y tres índices de malestar global.

Campbell y Stark (1990) utilizaron el SCL90-R, junto a otros instrumentos, para determinar las diferencias entre sujetos con problemas de abuso en distintas sustancias ( 34 sujetos con abuso de cocaina) respecto a la presencia de psicopatología y a las características de personalidad. El grupo de consumidores de cocaína es el que presenta mayores puntuaciones en las escalas de hostilidad, ideación paranoide y sen-

Tabla 1. Productos derivados del arbusto de la coca

\begin{tabular}{lcccc}
\hline \multicolumn{1}{c}{ Tipo sustancia } & Vía & $\begin{array}{c}\text { Inicio de los } \\
\text { efectos (seg.) }\end{array}$ & $\begin{array}{c}\text { Duración del } \\
\text { efecto (min.) }\end{array}$ & $\begin{array}{c}\text { Concentración } \\
\text { de cocaína }\end{array}$ \\
\hline Hoja de coca Mascada & Oral & $300-600$ & $45-90$ & $0,5-1,5 \%$ \\
Clorhidrato de cocaína & & $600-1800$ & - & $12-75 \%$ \\
Clorhidrato de cocaína & Intranasal & $120-180$ & $30-45$ & $10-20$ \\
Clorhidrato de cocaína & Intravenosa & $30-45$ & $5-10$ & $40-85 \%$ \\
Pasta de coca & Fumada & $8-10$ & $5-10$ & $30-85 \%$ \\
Crack de base libre & & $8-10$ & & \\
\hline
\end{tabular}

Fuente: Modificado de San (1996); Gold (1997); y Lizosaín, Moro y Lorenzo (2001). 
sibilidad interpersonal. Kleinman et al. (1990) lo utilizaron para evaluar la presencia de alteraciones en alguna de las nueve áreas en una muestra de consumidores de cocaina en el momento de entrada en tratamiento ( 76 sujetos con abuso de cocaína, el $73 \%$ consumidores de crack). Las puntuaciones de estos sujetos son superiores a las obtenidas en población normal, destacando las puntuaciones medias de las escalas de depresión, ideación paranoide y obsesión-compulsión.

En España destaca el estudio realizado por Pedrero, Puerta, Segura y Martínez (2004) que utilizan el SCL-90-R en un estudio transversal para analizar la evolución de los síntomas psicopatológicos en pacientes en tratamiento por abuso o dependencia de drogas. Destacan las puntuaciones medias obtenidas por los consumidores de cocaína en tratamiento en las escalas de depresión, obsesión-compulsión e ideación paranoide.

Por lo tanto, el SCL-90-R nos facilita obtener un perfil muy amplio de los síntomas piscopatológicos del paciente y que cuentan con un importante respaldo en el área de investigación de las drogodependencias. Sí tenemos que indicar que no permite establecer un diagnóstico específico a través de sus distintas escalas, por lo que hay que tener precaución con las interpretaciones que se hagan. Con él sabemos cual es el malestar que está padeciendo el paciente en la última semana, y por lo tanto contamos con una medida específica para evaluar la mejoría o empeoramiento percibido por el paciente a medida que avanza el tratamiento.

Con la realización del presente estudio nos planteamos los siguientes objetivos: (1) Evaluar con el SCL-90-R una muestra de personas con dependencia de la cocaína que están en tratamiento en centros de drogodependencias. (2) Comparar las puntuaciones medias obtenidas en cada una de las escalas, con las puntuaciones medias de la población normal. (3) Determinar si hay diferencias significativas en función de diversas variables sociodemográficas y características del consumo, en concreto: sexo, edad, edad del primer consumo de cocaína, años que lleva consumiendo cocaína, frecuen- cia en el consumo de cocaína en los seis meses previos al inicio del tratamiento, vía principal de consumo, tiempo de abstinencia en el consumo de cocaína, realización de tratamientos previos por consumo de drogas, si consumió heroina alguna vez en su vida, y la cantidad máxima de cocaína consumida en un solo día.

\section{MÉTODO}

\section{Participantes}

Participaron 115 personas, 99 hombres y 16 mujeres, que estaban en tratamiento en seis Centros públicos de Drogodependencias de Galicia. Fueron seleccionados de forma consecutiva entre el 23 de septiembre de 2003 y el 28 de abril de 2005. Los criterios de inclusión fueron: la cocaína es la droga principal que motiva el tratamiento, dependencia de la cocaína según criterios del DSM-IV-TR, y llevar un mínimo de tres semanas y un máximo de seis semanas abstinente en el consumo de cocaína. Los criterios de exclusión fueron: presencia de alteraciones psicóticas graves e incapacidad de responder a los instrumentos de evaluación. Inicialmente, fueron evaluados 119 sujetos, un sujeto no cumplía los criterios para el diagnóstico de dependencia de la cocaína, y tres superaban las seis semanas de abstinencia por lo que fueron eliminados del estudio.

\section{Instrumentos utilizados}

Entrevista Estructurada de DSM-IV (SCID) en su versión española (First, Spitzer, Gibbson, Williams y Smith-Benjamin, 1998), para evaluar la dependencia de la cocaína.

Para analizar las variables sociodemográficas y caracteristicas del consumo de cocaina elaboramos varios ítems que administramos al inicio de la evaluación. Evaluamos entre otros aspectos: sexo, edad, edad del primer consumo de cocaína, años que lleva consumiendo cocaína, frecuencia en el consumo de cocaina en los seis meses previos al inicio del tratamiento, vía principal de consumo, tiempo de abstinencia en 
el consumo de cocaína, realización de tratamientos previos por consumo de drogas, si consumió heroína alguna vez en su vida, y la cantidad máxima de cocaína consumida en un solo día.

Cuestionario de 90 Sintomas Revisado (SCL-90-R), en su versión española de Derogatis (2002). Evalúa el malestar subjetivo percibido por el sujeto a partir de 90 síntomas que se agrupan en nueve escalas (somatización, obsesión-compulsión, sensibilidad interpersonal, depresión, ansiedad, hostilidad, ansiedad fóbica, ideación paranoide y psicoticismo) y tres índices generales: Índice General Sintomático (IGS), Total de Síntomas Positivos (TSP) e Índice de Malestar (PSDI). El GSI evalúa la intensidad del sufrimiento psíquico y psicosomático global; el PST evalúa la amplitud y diversidad de la psicopatología a partir del número total de síntomas presentes; el PSDI indica la intensidad sintomática media, es decir, el estilo característico del individuo para experimentar el sufrimiento psíquico, si el sujeto maximiza o minimiza sus respuestas. El análisis de estos tres índices es uno de los aspectos más valorados de este cuestionario, ya que indican de forma general el malestar que tiene el paciente. El SCL-90$R$ evalúa en la versión española la existencia de tres niveles de morbilidad psiquiátrica: ausencia de morbilidad (población normal), presencia de morbilidad psiquiátrica menor generalmente asociada a trastornos funcionales o psicosomáticos (población temporo-mandibular), y presencia de patología psiquiátrica que se puede enmarcar en un diagnóstico psiquiátrico específico (población psiquiátrica) (Derogatis, 2002). Cuando el sujeto se sitúa en el centil 80 o por encima en el índice IGS o al menos en dos dimensiones sintomáticas en la tabla de población normal, se pasa a la tabla de pacientes con disfunción psicosomática temporo-mandibular; si las puntuaciones en esta tabla son superiores al centil 80 en mujeres o al 70 en hombres, en el GSI o en la menos dos escalas sintomáticas, se pasa a las tablas de población psiquiátrica. Por lo tanto, en función de la presencia e intensidad de los síntomas, el sujeto se clasifica en las tablas de población normal, temporo-mandibular o psiquiátrica.

\section{Procedimiento}

Los pacientes fueron seleccionados consecutivamente por los psicólogos de los Centros de Drogodependencias, entre septiembre de 2003 y abril de 2005. Si cumplían los criterios de inclusión en el estudio eran citados para ser evaluados por nosotros independientemente. Todos los sujetos firmaron el consentimiento informado para participar en el estudio.

\section{Tratamiento estadistico}

Se utilizó el paquete estadístico SPSS para Windows (versión 12.0). Se realizaron análisis de frecuencias y tablas de contingencia con el estadístico chi-cuadrado.

\section{RESULTADOS}

En el SCL-90-R las puntuaciones medias obtenidas son las que recogemos en la Tabla 2. Las más destacables (más elevadas) son las siguientes: en la escala de depresión la media es de 1,03 (DT =0,78), en la escala de obsesión compulsión la media es de $1,01(\mathrm{DT}=0,70)$, en la de ideación paranoide la media es de 1,00 (DT $=0,76)$, y en la escala de sensibilidad interpersonal la media es $0,87(\mathrm{DT}=0,70)$. En los indices generales las puntuaciones medias son las siguientes: en el índice general sintomático la puntuación media es de 0,83 (DT = 0,56 ), en el total de síntomas positivos la media es de 40,07 (DT $=18,83)$, y en el índice de malestar la media es de $1,79(\mathrm{DT}=0,55)$. Las puntuaciones medias obtenidas son en todas las escalas superiores a las medias del baremo de la población normal utilizados para validar este cuestionario en España (Derogatis, 2002).

En función de las puntuaciones que obtiene, cada sujeto se clasifica en una población (normal, temporo-mandibular o psiquiátrica) las 
Tabla 2. Puntuaciones medias obtenidas con el SCL-90-R

\begin{tabular}{|c|c|c|c|c|}
\hline & \multicolumn{2}{|c|}{ Muestra dependientes de la cocaína } & \multicolumn{2}{|c|}{ Baremos población norma } \\
\hline & Media & $D T$ & Media & $D T$ \\
\hline Somatización & 0,66 & 0,63 & 0,55 & 0,55 \\
\hline Obsesión-compulsión . & 1,01 & 0,70 & 0,60 & 0,51 \\
\hline Sensibilidad interpersonal & 0,87 & 0,73 & 0,45 & 0,44 \\
\hline Depresión $\ldots \ldots \ldots$ & 1,03 & 0,78 & 0,72 & 0,55 \\
\hline Ansiedad . & 0,76 & 0,74 & 0,52 & 0,49 \\
\hline Hostilidad . . . . . . . & 0,65 & 0,65 & 0,45 & 0,53 \\
\hline Ansiedad fóbica & 0,44 & 0,58 & 0,25 & 0,36 \\
\hline Ideación paranoide & 1,00 & 0,76 & 0,47 & 0,50 \\
\hline Psicoticismo & 0,65 & 0,65 & 0,21 & 0,30 \\
\hline Índice general sintomático $\ldots \ldots \ldots \ldots$ & 0,83 & 0,56 & 0,51 & 0,36 \\
\hline Total de síntomas positivos $\ldots \ldots \ldots \ldots$ & 40,07 & 18,83 & 25,30 & 14,30 \\
\hline Índice de malestar $\ldots \ldots \ldots \ldots \ldots \ldots$ & 1,79 & 0,55 & 1,75 & 0,48 \\
\hline
\end{tabular}

frecuencias de los sujetos clasificados en cada población son las siguientes: en la población normal se clasifica un $29,6 \%$ de la muestra $(n=34)$, en la población temporo-mandibular un $33,9 \%(n=39)$ y en la psiquiátrica un $36,5 \%$ de la muestra $(n=42)$.

Otro de los análisis realizados es transformar las puntuaciones directas obtenidas por los sujetos en centiles de la tabla de la población psiquiátrica. El objetivo es determinar el porcentaje de sujetos, en cada una de las escalas, que obtienen una puntuación por encima de la media de la población psiquiátrica (ver Tabla 3). Las escalas en las que un mayor porcentaje de personas con dependencia de la cocaína se sitúan por encima del centil 50 son la escala de hostilidad ( $41,7 \%$ por encima de la media), la de ideación paranoide $(41,7 \%)$, y la escala de psicoticismo $(37,4 \%)$. Respecto a los índices destaca el total de síntomas positivos, el $37,4 \%$ de los sujetos está por encima de la media de la población psiquiátrica.

Otro objetivo es determinar si hay diferencias significativas entre las puntuaciones en las escalas y en los índices generales transformadas en centiles en las tablas de población psiquiátrica, y diversas variables sociodemográficas (edad, sexo) y características del consumo de cocaína (edad del primer consumo de coca-

Tabla 3. Puntuaciones en el SCL-90-R igual o por debajo de la media y por encima de la media en la población psiquiátrica

\begin{tabular}{|c|c|c|c|c|}
\hline & \multicolumn{2}{|c|}{ Por debajo de la media } & \multicolumn{2}{|c|}{ Por encima de la media } \\
\hline & $n$ & $\%$ & $n$ & $\%$ \\
\hline Somatización $\ldots \ldots \ldots \ldots \ldots \ldots$ & 98 & 85,2 & 17 & 17,8 \\
\hline Obsesión-Compulsión $\ldots \ldots \ldots \ldots$ & 78 & 67,8 & 37 & 32,2 \\
\hline Sensibilidad Interpersonal $\ldots \ldots \ldots \ldots$ & 76 & 66,1 & 39 & 33,9 \\
\hline Depresión $\ldots \ldots \ldots \ldots \ldots \ldots \ldots$ & 89 & 77,4 & 26 & 22,6 \\
\hline Ansiedad $\ldots \ldots \ldots \ldots \ldots \ldots \ldots$ & 90 & 78,3 & 25 & 21,7 \\
\hline Hostilidad $\ldots \ldots \ldots \ldots \ldots \ldots \ldots$ & 67 & 58,3 & 48 & 41,7 \\
\hline Ansiedad Fóbica $\ldots \ldots \ldots \ldots \ldots \ldots$ & 86 & 74,8 & 29 & 25,2 \\
\hline Ideación Paranoide $\ldots \ldots \ldots \ldots \ldots$ & 67 & 58,3 & 48 & 41,7 \\
\hline Psicoticismo $\ldots \ldots \ldots \ldots \ldots \ldots$ & 72 & 62,6 & 43 & 37,4 \\
\hline Indice General Sintomático . . . . . . . & 86 & 74,8 & 29 & 25,2 \\
\hline Total de Síntomas Positivos . . . . . . . . & 72 & 62,6 & 43 & 37,4 \\
\hline Indice de Malestar $\ldots \ldots \ldots \ldots \ldots$ & 89 & 77,4 & 26 & 22,6 \\
\hline
\end{tabular}


ína, años que lleva consumiendo cocaína, frecuencia de consumo en los seis meses previos al inicio del tratamiento, vía principal de consumo, tiempo de abstinencia, consumo de heroina alguna vez en la vida y realización de tratamientos previos por problemas con el consumo de drogas y cantidad máxima de cocaína consumida en un solo día). Se encontraron diferencias significativas entre las siguientes variables: en la escala de obsesión-compulsión en función de la edad del sujeto, un $64,9 \%$ de los que tienen una puntuación por encima de la media en la población psiquiátrica tienen entre 18 y 30 años $\left(\chi_{(1)}^{2}=4,544, p<0,05\right)$. En la escala de ansiedad hay diferencias significativas en función de la edad del primer consumo de cocaina $\left(\chi_{(2)}^{2}=7,090, p<0,05\right)$, el $52 \%$ de los sujetos que tienen una puntuación por encima de la media en la población psiquiátrica empezaron a consumir cocaína cuando tenían entre 14 y 17 años, es decir la edad de inicio más temprana de la muestra. En la escala de hostilidad hay diferencias significativas en función del sexo y del número de años que llevan consumiendo. De los sujetos que están por encima de la media en la escala de hostilidad, el $93,8 \%$ son hombres $\left(\chi_{(1)}^{2}=4,039, p<0,05\right)$. En cuanto al número de años que llevan consumiendo cocaína un $22.9 \%$ de los que puntúan por encima de la media en la escala de hostilidad llevan consumiendo entre 11 y 15 años, y otro $22,9 \%$ llevan de 16 años en adelante $\left(\chi_{(3)}^{2}=9,394\right.$, $\mathrm{p}<0,05$ ).

En la relación entre la cantidad máxima de cocaína que han consumido en un día y el SCL90-R (entre 1,0 y 2,0 gramos, entre 2,1 y 5,0 gramos o más de 5,0 gramos), destaca el porcentaje de sujetos que han consumido más de 5,0 gramos en un día, ya que obtuvieron puntuaciones por encima de la media de la población psiquiátrica en las escalas de sensibilidad interpersonal $\left(\chi_{(2)}^{2}=7,651, \mathrm{p}<0,05\right)$, ansiedad $\left(\chi_{(2)}^{2}=16,705, \mathrm{p}<0,001\right)$, hostilidad $\left(\chi_{(2)}^{2}=\right.$ $7,294, \mathrm{p}<0,05)$, ansiedad fóbica $\left(\chi_{(2)}^{2}=8,855\right.$, $\mathrm{p}<0,05)$, ideación paranoide $\left(\chi_{(2)}^{2}=8,599, \mathrm{p}\right.$ $<0,05)$, psicoticismo $\left(\chi_{(2)}^{2}=11,345, \mathrm{p}<0,01\right)$ $\mathrm{y}$ el índice general sintomático $\left(\chi_{(2)}^{2}=13,539\right.$, $\mathrm{p}<0,01)$.

\section{DISCUSIÓN}

Evaluamos una muestra de personas con dependencia de la cocaina que demandan tratamiento en centros de drogodependencias con el SCL-90-R. Las escalas de sintomas con una puntuación media más alta son las de depresión $(1,03)$, obsesión-compulsión $(1,01)$, ideación paranoide $(1,0)$ y sensibilidad interpersonal $(0,87)$.

Los estudios en los que contamos con las puntuaciones medias de consumidores de cocaína en las distintas escalas del SCL-90-R (Campbell y Stark, 1990; Kleinman et al., 1990; Pedrero et al., 2004) señalan que las escalas con mayores puntuaciones medias son la escala de depresión, la escala de ideación paranoide, la escala de obsesión-compulsión y la de sensibilidad interpersonal. Los estudios revisados que utilizan el SCL-90-R, o su versión abreviada el BSI, para evaluar consumidores de cocaína, coinciden en señalar como más destacadas las escalas de ideación paranoide (Campbell y Stark, 1990; Pedrero et al., 2004; Sánchez-Hervás et al., 2000; SánchezHervás et al., 2001), hostilidad (Campbell y Stark, 1990; Pedrero et al., 2004; Sánchez-Hervás et al., 2000; Sánchez-Hervás et al., 2001), obsesión-compulsión (Campbell y Stark, 1990; Sánchez-Hervás et al., 2001), y depresión (Sánchez-Hervás et al., 2000; Sánchez-Hervás et al., 2001).

Por lo tanto, los resultados que hemos obtenido coinciden con estudios realizados previamente con consumidores de cocaína (abuso / dependencia).

En el estudio de Pedrero et al. (2004) también utilizaron el SCL-90-R con una muestra de consumidores de heroína en tratamiento. Las escalas en las que tienen una puntuación media más alta son la de depresión $(1,51)$, la de obsesión-compulsión $(1,24)$ y la de somatización $(1,12)$. Las de depresión y de obsesión-compulsión son también las escalas de síntomas en las que nuestra muestra de personas dependientes de la cocaína obtienen una puntuación media más alta, pero en la escala de somatización la puntuación es muy inferior $(0,66)$. 
Cuando comparamos las puntuaciones medias obtenidas por esta muestra de personas dependientes de la cocaína con las puntuaciones medias de población normal que se utilizaron como baremos en la validación del SCL90-R en España (Derogatis, 2002), observamos que las personas con dependencia de la cocaína tienen puntuaciones medias superiores en todas las escalas del SCL-90-R. Las escalas en las que hay una mayor diferencia entre dependientes de la cocaína y población normal son las de ideación paranoide, psicoticismo y obsesión-compulsión.

Respecto a la clasificación de los sujetos en función de las tres tablas de baremos del SCL90-R (normal, temporo-mandibular y psiquiátrica), hay un $36,5 \%$ que se clasifica en la población psiquiátrica. Es decir, el malestar percibido por estos sujetos en el momento de ser evaluados con el SCL-90-R es similar al de la población psiquiátrica en uno de cada tres.

Respecto a la existencia de diferencias significativas en función de diversas variables sociodemográficas y características del consumo, la variable más relevante que aparece es la cantidad máxima de cocaína consumida en un día. Es más probable que los sujetos que han consumido más de cinco gramos de cocaína en un dia tengan puntuaciones por encima de la media de la población psiquiátrica en las escalas de sensibilidad interpersonal, ansiedad, ansiedad fóbica, hostilidad, ideación paranoide, psicoticismo y el índice general sintomático. Por lo tanto, consumir importantes cantidades de cocaína en poco tiempo está relacionado con un mayor malestar percibido por el sujeto y con la presencia de un mayor número de síntomas de hostilidad, ansiedad, psicoticismo e ideación paranoide, que los sujetos que no han realizado consumos tan altos de cocaína.

Los anteriores resultados son de gran relevancia cara a la puesta en práctica del tratamiento de las personas con problemas con el consumo de drogas que lo demandan. Cuando una persona acude a un centro de drogodependencias demandando tratamiento, es con frecuencia porque presenta un nivel de malestar derivado de dicho consumo (Barrio-Anta et al., 1997; Bobes et al., 2001; Muga, 2001), de ahí la explicación de que la puntuación media de estos sujetos sea superior a la media de la población normal. En muchos casos este malestar va a disminuir con la intervención terapéutica sobre el problema con el consumo de drogas, pero en otros puede precisar una intervención específica. Si las puntuaciones son muy altas en alguna de las escalas de síntomas o si a medida que avanza el tratamiento se observa que no hay una mejoría en los síntomas psicopatológicos que presenta la persona, es preciso realizar una evaluación más especifica de dichos síntomas y diseñar una intervención sobre los mismos. La presencia de problemas psicopatológicos puede interferir en el tratamiento del problema con el consumo de drogas, por lo que siempre es necesario tenerlos en cuenta.

En el futuro deberian de seguir realizándose estudios con personas con dependencia de la cocaína, ya que la demanda de tratamiento sigue aumentando tanto en España como en otros paises y todavía siguen siendo pocos los estudios publicados en nuestro país que utilizan muestras de personas con diagnóstico de dependencia de la cocaína. Respecto a la utilización de cuestionarios como el SCL-90-R, próximas investigaciones podrian ir dirigidas a efectuar seguimientos de las personas con dependencia de la cocaína, ya que es un cuestionario sencillo de administrar y que permite valorar la evolución de las personas en tratamiento.

\section{REFERENCIAS}

Barrio-Anta, G., López-Gigosos, R., de la Fuente de Hoz, L., y Rodríguez-Artalejo, F. (1997). Patrones de uso de la cocaína en un grupo de consumidores de esta droga sin consumo de heroína captados fuera de los servicios asistenciales. Medicina Clínica, 10, 364-369.

Bobes, J., Sáiz, P.A., González, M.P., y Bascarán, M.T. (2001). Epidemiología del uso/abuso de cocaina. Adicciones, 13 (Supl. 2), 23-36.

Brown, T.G., Seraganian; P: y Tremblay, J. (1994). Alcoholics also dependent on cocaine in treatment: do they different from «pure» alcoholics. Addictive Behaviors, 19, 105-112.

Campbell, B.K. y Stark, M. J. (1990). Psychopathology and personality characteristics in different 
forms of substance abuse. International Journal of the Addictions, 25, 1467-1474.

Castaneda, R. (1994). Empirical assessment of the selfmedication hypothesis among dually diagnosed inpatients. Comprehensive Psychiatry, 35, 180-184.

Castaneda, R., Galanter, M. y Franco, H. (1989). Self- medication among addicts with primary psychiatric disorders. Comprehensive Psychiatry, 30, 80-83.

Derogatis, L.R. (1977). The SCL-90-R. Baltimore: Clinical Psychometric Research.

Derogatis, L.R. (2002). SCL-90-R. Cuestionario de 90 sintomas revisado. Manual. Madrid: Tea Ediciones.

Derogatis, L.R., Lipman, R.S., y Covi, L. (1973). The SCL-90: An outpatient psychiatric rating scale. Psychopharmacology Bulletin, 9, 13-28.

First, M.B. y Gladis, M. M. (1996). Diagnóstico y diagnóstico diferencial de los trastornos psiquiátricos y por uso de sustancias. En J. Solomon, S. Zimberg y E. Shollar (Eds.), Diagnóstico dual (pp. 41-56). Barcelona: Ediciones Neurociencias.

First, M.B., Spitzer, R.L., Gibbson, M., Williams, J.B.W. y Smith-Benjamin, L. (1998). Guía del usuario de la entrevista clínica estructurada para los trastornos del eje I del DSM-IV-SCID-I. Barcelona: Masson.

García, J.C. (2002). De la coca a la cocaina. Una historia por comprender. México D.F.: Ediciones del Milenio.

Gawin, F. H. y Kleber H. D. (1986). Abstinence symptomatology and psychiatric diagnosis in cocaine abusers. Archives of General Psychiatry, 43, 107-113.

Gold, M.S. (1997). Cocaina. Barcelona: Ediciones Neurociencias.

Kleinman, P.H., Kang, S., Lipton, D.S., Woody, G.E., Kemp, J. y Millman, R.B. (1992). Retention of cocaine abusers in outpatient psychotherapy. American Journal of Drug and Alcohol Abuse, 18, 29-43.

Kleinman, P.H., Miller, A.B., Millman, R.B., Woody, G.E., Todd, T., Kemp, J. y Lipton, D.S. (1990). Psychopathology among cocaine abusers entering treatment. Journal of Nervous and Mental Disease, 178, 442-447.

Lizasoain, I., Moro, M. A. y Lorenzo, P. (2001). Cocaína: aspectos farmacológicos. Adicciones, 13 (sup. 2), 37-45.

Muga, R. (2001). Y ahora la cocaína. Medicina Clinica, 117, 584-585.

Observatorio Europeo de la Droga y las Toxicomanías (2004). Informe anual 2004 del Observatorio Europeo de la Droga y la Toxicomanía (OEDT) sobre el problema de la drogodependen- cia en la Unión Europea y Noruega. Recogido el 13 de Enero de 2005 en http://www. mir.es/pnd/ coopera/pdf/informeOEDT_2004.pdf.

Ochoa, E. (2000). Cocaína y comorbilidad psiquiátrica. Actas Españolas de Psiquiatria, 28, 40-52.

Pedrero, E.J., Puerta, C., Segura, I. y Martínez, S. (2004). Evolución de la sintomatología psicopatológica de los drogodependientes a lo largo del tratamiento. Trastornos Adictivos, 6, 175-191.

Plan Nacional sobre Drogas (2006). Observatorio Español sobre drogas. Informe 2004

Recogido el 23 de enero de 2006 en: http://www. pnd.msc.es/Categoria2/publica/pdf/oed-2004.pdf

Rose, M.R., Brown, B.S. y Haertzen, Ch.A. (1989). Comparison of the characteristics and functioning of cocaine treatment and cocaine research subjects. American Journal of Drug and Alcohol Abuse, 15, 251-260.

Rosenthal, R.N. y Westreich, L. (1999). Treatment of persons with diagnoses of substance use disorders and other psychological problems. En B.S. McCrady y E.E. Epstein (Eds.), Addictions. A comprehensive guidebook (pp. 105-120). New York: Oxford University Press.

San, L. (1996). Dependencia de la cocaina. En: Becoña, E., Rodríguez, y Salazar, I. (Coord.), Drogodependencias III. Drogas ilegales (41-99). Santiago de Compostela: Servicio de publicacións da Universidade de Santiago de Compostela.

Sánchez, E. (2001). Trastornos adictivos y otros trastornos mentales. Psiquiatría Biológica, 8, 64-73.

Sánchez, E., Tomás, V. y Morales, E. (2000). Evaluación psicopatológica de pacientes dependientes de la cocaína. Atención Primaria, 26, 319-322.

Sánchez-Hervás, E., Gradolí, V. y Morales, E. (2001). Evaluación psicopatológica en dependientes al alcohol, heroína y cocaína mediante el Brief Symptom Inventory. Adicciones, 1, 61-66.

Swendsen, J.y Merikangas, K. (2000), The comorbidity of depression and substance use disorders. Clinical Psychology Review, 20, 173-189.

Weiss, R.D., Mirin, S.M., Griffin, M.L. y Michael, J.L. (1988). Psychopathology in cocaine abusers. Journal of Nervous and Mental Disease, 176, 719-725.

Weiss, R.D., Mirin, S. T., Michael, J. L. y Sollogub, A. C. (1986). Psychopathology in chronic cocaine abusers. American Journal of Drug and Alcohol Abuse, 12, 17-29.

Ziedonis, D. M. (1992). Comorbid psychopathology and cocaine addiction. En: T. R. Kosten y H. D. Kleber (Eds.), Clinician's guide to cocaine addiction. Theory, research and treatment (335-358). New York: The Guildford Press. 